\title{
Urea-based gemini surfactants: Synthesis, aggregation behaviour and biological activity
}

Martin Pisárčik ${ }^{\mathrm{a}^{*}}$, Matúš Pupák ${ }^{\mathrm{a}}$, Ferdinand Devínsky ${ }^{\mathrm{a}}$, László Almásy ${ }^{\mathrm{b}}$, Qiang Tian ${ }^{\mathrm{b}, \mathrm{c}}$, Marián Bukovskýd

${ }^{a}$ Department of Chemical Theory of Drugs, Faculty of Pharmacy, Comenius University, Bratislava, SK-83232 Slovakia

${ }^{b}$ Institute for Solid State Physics and Optics, Wigner Research Centre for Physics, Hungarian Academy of Sciences, H-1121 Budapest, Hungary

${ }^{c}$ Key Laboratory of Neutron Physics and Institute of Nuclear Physics and Chemistry, China Academy of Engineering Physics, Mianyang 621999, China

${ }^{d}$ Department of Cell and Molecular Biology of Drugs, Faculty of Pharmacy, Comenius University, Bratislava, SK-83232 Slovakia

\footnotetext{
*Corresponding author, Email: pisarcik@fpharm.uniba.sk, Tel: +421-2-50117329
} 


\begin{abstract}
Synthesis, aggregation properties in aqueous solution and antimicrobial activity of novel urea-based cationic gemini surfactants $N, N^{\prime}-$ bis $\{2-$

[(undecylkarbamoyl)amino] ethyl $\}-N, N, N^{\prime}, N^{\prime}$-tetramethylalkane- $\alpha, \omega$ -

diammoniumdibromides of variable spacer length are presented as a function of surfactant molecular structure. Utilizing the experimental methods of tensiometry, dynamic light scattering, time-resolved fluorescence quenching, neutron scattering, and zeta potential, the obtained data indicate a stronger aggregation tendency of urea-based surfactants with a short spacer composed of $2 \mathrm{CH}_{2}$ groups which was confirmed by all applied experimental methods. As results from the comparison with the gemini surfactants without urea groups in the alkyl tails and polymethylene spacer, the presence of urea groups enhances the aggregation properties of gemini molecules through additional hydrogen bonding interactions. A simple molecular model of the hydrogen bonding interaction between neighbouring urea-based gemini molecules is provided. For longer spacers of 4 to $10 \mathrm{CH}_{2}$ groups, the existence of monodisperse spherical micelles has been confirmed. The dependence of antimicrobial activity vs. spacer length shows nonlinear parabolic behaviour with the maximum antimicrobial activity found at the spacer length of $6 \mathrm{CH}_{2}$ groups for bacteria and $8 \mathrm{CH}_{2}$ groups for fungi. This is related to a different composition of cell membrane of the used microorganisms.
\end{abstract}

Keywords: Urea; Gemini surfactant; Surfactant spacer; Hydrogen bonding; Micelle aggregation number 


\section{Introduction}

Urea affects the physical and aggregation properties of proteins and surfactants in a broad way. It controls folding and unfolding of proteins [1,2], enhances solubility of hydrocarbons in water and inhibits aggregation of surfactants. Especially, the area of interactions between urea and surfactant molecules attract a significant research interest. The urea headgroup interactions within the surfactant molecule structure control some of the physical properties of urea-based surfactants (melting point, solubility) [3]. Urea inhibits aggregation of surfactant molecules, as documented by the critical micelle concentration $(\mathrm{cmc})$ increase, micelle hydrodynamic size and aggregation number decrease of dioctylsuccinate [4], sodium dodecyl sulfate, cetylpyridinium bromide and cetyltrimethylammonium bromide micelles [5-7]. The addition of urea slightly decreases the micropolarity and strongly increases the microviscosity of the micellar interface of sodium dodecylbenzenesulfonate micelles indicating a possible replacement of some water molecules by urea [8]. Urea also affects the micellar structure of double chain gemini surfactants. A complex effect of urea on gemini surfactant micelles was found for gemini surfactants with 2 methylene groups in the spacer and dodecyl, tetradecyl a hexadecyl chains. While inhibiting the aggregation properties of gemini surfactants with 2 methylene groups in the spacer and dodecyl chains ( $\mathrm{cmc}$ increase, micellar size decrease), the presence of urea favours the aggregation of gemini surfactants with longer alkyl chains [9]. Urea significantly affects the aggregation properties and microstructure of nonionic micelles consisted of triblock copolymers [10] or sugar-based surfactants (glucopyranosides and maltopyranosides) [11]. The increased aggregation behaviour (vesicles formation, organogelators) was confirmed for fluorinated amphiphilic urea derivatives $[12,13]$. Single chain and double chain urea-based anionic surfactants $(\beta-$ alanine derivatives) [14] and anionic gemini surfactants derived from cysteine [15] showed better tendency to adsorb at the air/water interface than to form micelles. Urea-based gemini surfactants with octyl chains displayed cmc values comparable to other carboxylate surfactants of higher hydrocarbon chain length (decyl and dodecyl chains) [15] which is attributed to the hydrogen bonding ability of urea group [16]. Increase of micelle ionization degree and decrease in anion selectivity of cetyltrimethylammonium bromide and cetyltrimethylammonium chloride micelles in presence of urea indicates a preferential headgroup solvation by urea squeezing the counterions out of the Stern layer [17]. 
Double chain gemini surfactants continue to attract the scientific interest due to their outstanding aggregation properties which are superior to those of conventional single chain surfactants. Their unusual physical and aggregation properties make them suitable for a broad range of applications. cmc of gemini surfactants being 10 to 100-times smaller than that of conventional single-chain surfactants, results in much less skin irritation which allows to use them as skin permeation enhancers [18]. A smaller value of their cmc also enables them to be efficient solubilisers of low soluble drugs [19], dyes [20,21], hydrocarbons such as n-hexane and toluene [22]. Gemini surfactants can also serve as structural units in the formulation of cationic liposomes [23]. As for industrial applications, gemini surfactants show inhibitive effect on iron corrosion [24], act as efficient agents in oil recovery [25], styrene polymerisation [26,27], dying of textiles [28,29]. They appeared to be good stabilisers of polymer latex particles [30], separation agents in capillary chromatography [31], gelators of organic solvents [32], and reactants in the synthesis of silica mesophases [33]. A positive environmental impact of gemini surfactants is, apart from their low quantities used for a given application, to relate to the presence of biodegradable groups in their molecular structure such as carbon-carbon double bond [34] or carbonate linkages [35].

Cationic bisammonium gemini surfactants with polymethylene spacer - alkanediyl$\alpha, \omega$-bis(alkyldimethylammonium) dibromides and their derivatives with various functional groups in the spacer have been broadly studied in the literature. Their physical and aggregation characteristics such as critical micelle concentration and surface properties [36-41], micelle aggregation number and size [42-44], antimicrobial activity [45], aggregate structure variability in aqueous solutions as a function of the spacer length [46], indicate unique properties and aggregation behaviour of this class of surfactants which predetermines them to be used in a broad range of applications.

Within this work, we provide a complete view of a series of novel urea-based gemini surfactants from the viewpoint of synthesis, physico-chemical and aggregation properties (tensiometry, dynamic light scattering, time-resolved fluorescence quenching, neutron scattering and zeta potential measurements) of aggregates of these surfactants as well as report some of their biological activities.

\section{Experimental}


Gemini surfactants $N, N^{\prime}$-bis $\left\{2-\left[(\right.\right.$ undecylkarbamoyl)amino]ethyl $\}-N, N, N^{\prime}, N^{\prime}-$ tetramethylalkane- $\alpha, \omega$-diammoniumdibromides with urea fragments in the alkyl chains were synthesized with a polymethylene spacer length s ranging from 2 to $10 \mathrm{CH}_{2}$ groups. Urea-based surfactants are referred as u2 $(\mathrm{s}=2), \mathbf{u} 4(\mathrm{~s}=4), \mathbf{u} 6(\mathrm{~s}=6), \mathbf{u} 8(\mathrm{~s}=8)$, and $\mathbf{u 1 0}$ $(\mathrm{s}=10)$. Their molecular composition is shown in Fig 1 .

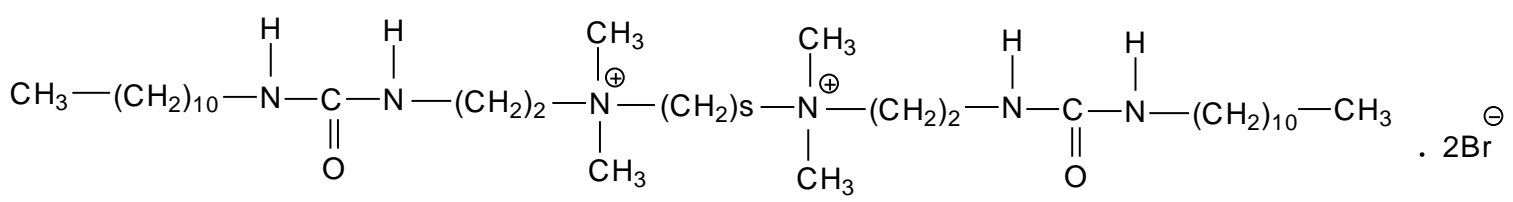

$$
\mathrm{s}=2,4,6,8,10
$$

Fig. 1. Molecular structure of urea-based surfactants

\subsubsection{Preparation of 1-[2-(dimethylamino) ethyl]-3-undecylurea}

The 1-isocyanatoundecane was prepared according to [47] and was freshly distilled before use (b. p. $143^{\circ} \mathrm{C}$ at $3.2 \mathrm{kPa}$, [47] gives b. p. at $103^{\circ} \mathrm{C}$ at $0.4 \mathrm{kPa}$ ). The $1-[2-$ (dimethylamino)ethyl]-3-undecylurea was prepared using a similar procedure as was used for the 1-cyclohexyl-3-[2-(dimethylamino)ethyl] urea [48] as follows. Into the ice-cooled stirred solution of 1-isocyanotoundecane (10 g, $35 \mathrm{mmol})$ in $25 \mathrm{~mL}$ of dry acetonitrile, 2.1 $\mathrm{g}(24 \mathrm{mmol})$ of 2-( $\mathrm{N}, \mathrm{N}$-dimethyl)ethane-1,2-diamine were added dropwise during 15 minutes. After the whole portion of the amine had been added, the solution was stirred for one hour and the temperature was maintained between -5 and $0^{\circ} \mathrm{C}$. The crude residue was filtered and washed with anhydrous diethyl ether. The crude product was dried in vacuum and was used in the next step without further purification. The NMR and spectroscopic data are shown in the Supplementary material.

\subsubsection{Preparation of gemini surfactants}

$\mathbf{u} 2$ to $\mathbf{u 1 0}$ were obtained via $\mathrm{S}_{N} 2$ Menshutkin reaction as follows: 1 eq. of 1-[2(dimethylamino)ethyl]-3-undecylurea was suspended in $20 \mathrm{ml}$ of anhydrous acetonitrile and 0.51 eq. of corresponding $\alpha, \omega$-dibromoalkane was added and the mixture was refluxed for 24 hours in anhydrous conditions. After cooling the mixtures to the room 
temperature, the solvent was evaporated in vacuum and the crude residue was crystallized from anhydrous acetone. The final products were obtained as white hygroscopic powders which were dried in vacuum desiccator using $\mathrm{P}_{4} \mathrm{O}_{10}$. The NMR, spectroscopic and elemental analysis data are shown in the Supplementary material.

All the chemicals and solvents used were obtained from Fluka and were used without further purification. Anhydrous solvents, if used, were prepared using the standard laboratory procedures. The IR spectra were recorded on Nicolet FT IR 6700 spectrophotometer, the ${ }^{1} \mathrm{H}$ and ${ }^{13} \mathrm{C}$ NMR spectra were recorded on the Varian Mercury Plus spectrometer operating at a proton ${ }^{1} \mathrm{H}$ NMR frequency of $300 \mathrm{MHz}$ and at a carbon ${ }^{13} \mathrm{C}$ NMR frequency of $75.5 \mathrm{MHz}$. For protons, 32 scans per sample were recorded with the following parameters: pulse width $(\mathrm{PW})=5.0 \mu \mathrm{s}\left(45^{\circ}\right)$ and relaxation delay $(\mathrm{RD})=1.0 \mathrm{~s}$. For carbons, 4000 scans per sample were recorded. The FIDs were Fourier transformed. The spectra were referenced to the residual solvents signal of the $\mathrm{CDCl}_{3}(7.26 \mathrm{ppm})$. The values of chemical shifts are given in ppm and the values of the interaction constants $(J)$ are given in Hz. The tetramethylsilane (TMS, $0.01 \%$, v/v) was used as an internal standard.

The elemental analyses were performed using a CHNOS elemental analyser (Thermo Scientific). The melting points were determined using a Kofler micro hot stage. The measured values are reported uncorrected.

\subsection{Surface tension}

Surface tension of surfactant solutions was measured by using a Kruss K100MK2 tensiometer with the Wilhelmy plate method. The automated dosing of surfactant stock solution volumes into the solvent in the measurement vessel was realized by a computercontrolled Metrohm 765 Dosimat titrator dosing unit based on a pre-generated table of dosed stock surfactant solution volumes. The automated measurement process was controlled by a Kruss Laboratory Desktop control software. The data were recorded after the surface tension equilibrium value had been reached. The measurements were carried out at $25^{\circ} \mathrm{C}$. The surface excess $\Pi$ is related to the experimentally determined slope of the surface tension vs. log surfactant concentration dependence in the premicellar region $(\mathrm{d} \gamma / \mathrm{d} \log \mathrm{c})_{\mathrm{p}, \mathrm{T}}$ using the Gibbs adsorption equation [49] 


$$
\Pi=-\frac{1}{2.303 \text { i R T }}\left(\frac{\mathrm{d} \gamma}{\mathrm{d} \log \mathrm{c}}\right)_{\mathrm{p}, \mathrm{T}}
$$

$\Pi$ is expressed in $\mathrm{mol} / 1000 \mathrm{~m}^{2},(\mathrm{~d} \gamma / \mathrm{dlog} \mathrm{c})_{\mathrm{p}, \mathrm{T}}$ is expressed in $\mathrm{mN} / \mathrm{m}, \mathrm{R}=8.31 \mathrm{~J} \mathrm{~K}^{-1} \mathrm{~mol}^{-1}$ and $\mathrm{T}=298.15 \mathrm{~K}$. The value of the prefactor $\mathrm{i}=3$ which applies to ionic gemini surfactants, was used in the equation (1). The value $i=3$ is applied for the premicellar region of gemini surfactant concentrations where the gemini surfactant is completely ionized in the solution from which adsorption occurs [50]. The area per surfactant molecule A in $\mathrm{nm}^{2}$ was calculated from the surface excess $\Pi$ as follows [29]

$$
\mathrm{A}=\frac{10^{21}}{\Pi \mathrm{N}_{\mathrm{A}}}
$$

where $\mathrm{N}_{\mathrm{A}}$ is the Avogadro's number.

\subsection{Dynamic light scattering}

A Brookhaven light scattering system BI 9000, goniometer SM200 and an argon laser (the applied wavelength $514.5 \mathrm{~nm}$ ) were used for the dynamic light scattering measurements. The scattered intensity was registered at $90^{\circ}$ and at the temperature of $25^{\circ} \mathrm{C}$. Surfactant solutions for the light scattering measurements were prepared using deionized water and filtered for mechanical impurities through syringe filters with $0.45 \mu \mathrm{m}$ pore size. For each surfactant $\mathbf{u}(\mathbf{s})$ and each concentration c/cmc, 5 independent measurements of the autocorrelation function were recorded. $\mathbf{u}(\mathbf{s})$ micelle diameters were obtained from the particle size distributions which resulted from the application of the constrained regularized algorithm CONTIN [51] on the autocorrelation function.

\subsection{Zeta potential}

Zeta potential measurements were performed with the Brookhaven BI ZetaPlus equipment which is based on the measurement of the electrophoretic mobility utilizing the Doppler frequency shift. Zeta potential values were calculated from the measured mobility using the Smoluchowski limit for the mobility vs. zeta potential relationship. The mean value was calculated from a statistical set of 10 zeta potential recordings per each surfactant concentration. The measurements were taken at $25^{\circ} \mathrm{C}$. 


\subsection{Time-resolved fluorescence quenching}

The fluorescence decay curves were recorded using a lifetime fluorescence spectrometer LifeSpec II (Edinburgh Instruments). The spectrometer was operating at a $381 \mathrm{~nm}$ emission wavelength and the decay curves were fitted to eq. (3) [52]

$$
\mathrm{I}(\mathrm{t})=\mathrm{I}(0) \exp \left\{-\frac{\mathrm{t}}{\tau}-\mathrm{R}\left[1-\exp \left(-\frac{\mathrm{t}}{\tau_{\mathrm{Q}}}\right)\right]\right\}
$$

using a nonlinear weighted least squares procedure (the Edinburgh Instruments FAST software package with the micellar quenching analysis software module). $\mathrm{I}(\mathrm{t})$ and $\mathrm{I}(0)$ are the fluorescence intensities at time $\mathrm{t}$ and zero time, respectively. $\tau_{0}, \tau_{\mathrm{Q}}, \mathrm{R}$ are nonlinear fitting parameters where $\mathrm{k}_{\mathrm{Q}}=1 / \tau_{\mathrm{Q}}$ is the rate constant for fluorescence agent quenched by a quencher in a micellar solution. The micelle aggregation $\mathrm{N}$ number is related to the fitting constant $\mathrm{R}$ and the concentration of surfactant and quencher as follows [52]

$$
\mathrm{N}=\mathrm{R} \frac{\mathrm{c}-\mathrm{cmc}}{\mathrm{c}_{\mathrm{Q}}}
$$

$\mathrm{c}_{\mathrm{Q}}$ is the quencher concentration and $\mathrm{c}$ is the surfactant molar concentration. In the fluorescence quenching experiments, pyrene was used as a fluorescence agent and cetylpyridinium chloride as a quencher. The pyrene concentration was kept at a low level, such that the ratio pyrene concentration/concentration of surfactant micelles in the solution $<0.05$. The concentration ratio quencher concentration/concentration of surfactant micelles was close to 1 so that the quencher distribution is approximately one quencher molecule per micelle. The surfactant concentrations used were high enough to ensure that pyrene and quencher were completely solubilized in the micelles. All measurements were performed at $25^{\circ} \mathrm{C}$.

\subsection{Small angle neutron scattering}

SANS measurements were performed on the Yellow Submarine instrument installed at the cold neutron beamline of the 10MW steady-state reactor of the Budapest Neutron Centre (BNC), Hungary. The monochromatic beam with a mean wavelength of $0.43 \mathrm{~nm}$ and $30 \%$ FWHM was produced by a multidisc mechanical velocity selector. Gas filled multiwire detector of $64 * 64 \mathrm{~cm}^{2}$ sensitive area was placed at the distances of 1.3 and $5.5 \mathrm{~m}$ from the sample, to cover the $q$-interval of $0.12-3.8 \mathrm{~nm}^{-1}$. The measurements were 
performed at $25^{\circ} \mathrm{C}$. The sample solutions were filled in optical quartz glass cells with 5 $\mathrm{mm}$ path length. After the raw data treatment by standard procedures, the scattering curves have been analysed by a conventional model of charged micelles. For a dilute dispersion of identical objects in a homogeneous medium, e.g. colloidal or micellar solutions, the scattering intensity can be expressed as a product of the form factor of the particles, and the structure factor which describes the spatial arrangement of the particles.

$$
\mathrm{I}(\mathrm{q})=\mathrm{A} P(\mathrm{q}) \mathrm{S}(\mathrm{q})+\mathrm{bg}
$$

$\mathrm{P}(\mathrm{q})$, the micelle form factor was taken as an ellipsoid of rotation. A possible size polydispersity has not been included, as it would strongly correlate with the ellipsoid axis ratio. The structure factor $\mathrm{S}(\mathrm{q})$, was modelled by the rescaled mean spherical approximation for dilute charged colloidal dispersions, in which the micelles consist of partly ionized surfactant molecules and interact via screened Coulomb forces [53]. The background term "bg" accounts for the incoherent scattering which is angle-independent in the studied $q$-range and arises mostly from the scattering of hydrogen and deuterium atoms present in the samples. Finally, the theoretical equation is convoluted with the instrumental resolution function which was calculated for pinhole scattering geometry and finite wavelength resolution [54]. The least squares fitting was performed by SasView 3.0 software.

\subsection{Determination of antimicrobial activity}

The antimicrobial activity of $\mathbf{u ( s )}$ was evaluated in vitro as the minimum bactericidal concentration (MBC). It was determined using the standard broth dilution method [55]. The following representative groups of gram-positive and gram-negative bacteria were selected for the experiments: Staphylococcus aureus ATCC 6538, Escherichia coli ATCC 11229 and Candida albicans CCM 8186. Two commercially used quaternary ammonium disinfectants, cetylpyridinium bromide (CPB) and benzyldodecyldimethylammonium bromide (BDDAB), were used as the reference compounds. Their MBC values were determined and the method of testing applied within this experimental work is described in the papers $[55,56]$. 


\section{Results and discussion}

\subsection{Determination of $\mathrm{cmc}$ and interfacial area}

In Fig. 1 and 2, dependences of surface tension vs. log surfactant concentration are shown.

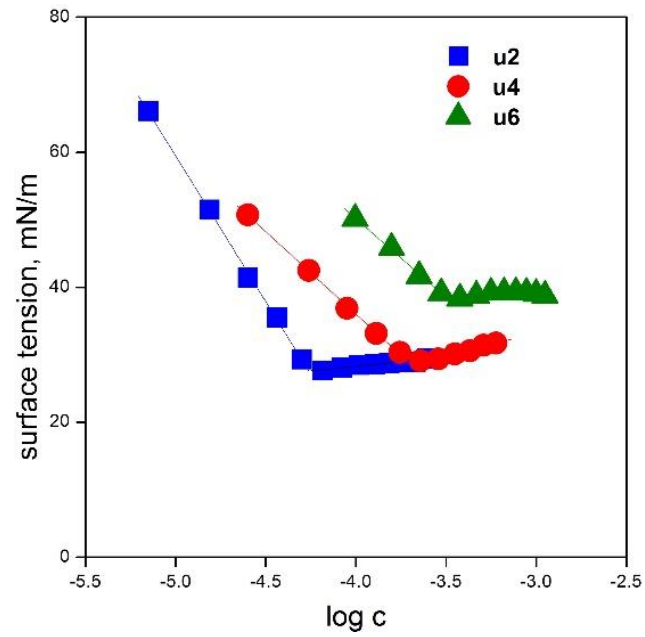

Fig. 1. Dependence of surface tension on log surfactant concentration for gemini surfactants $\mathbf{u} 2, \mathbf{u 4}, \mathbf{u 6}$.

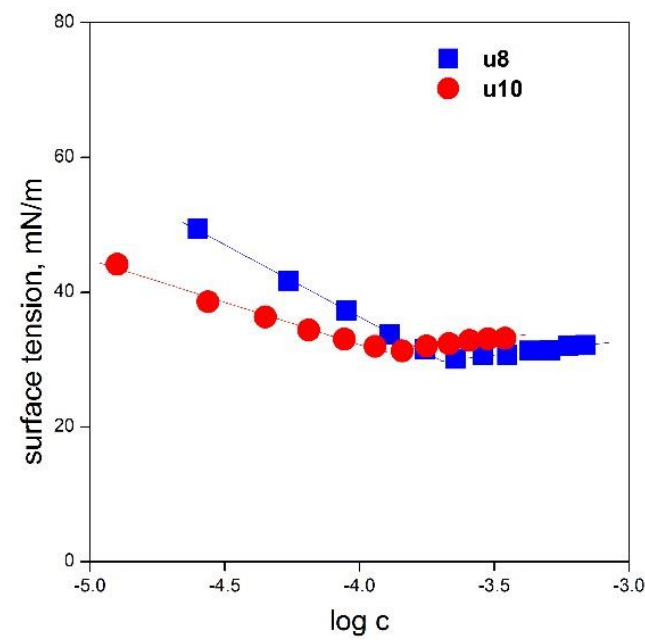

Fig. 2. Dependence of surface tension on log surfactant concentration for gemini surfactants $\mathbf{u} 8, \mathbf{u 1 0}$.

The $\mathrm{cmc}$ values were calculated as the intersection of the regression lines in the premicellar and micellar regions and are plotted in Fig. 3. 


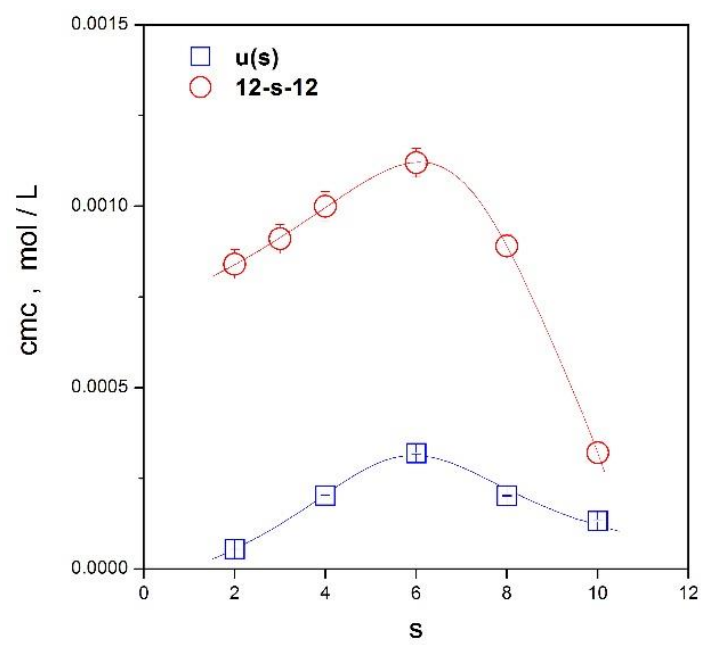

Fig. 3. cmc plotted as a function of $\mathbf{u}(\mathbf{s})$ gemini surfactant spacer length s. cmc of 12 -s-12 gemini surfactants [39] is indicated by open circles.

As follows from Fig. 3, the cmc dependence on the spacer length $\mathrm{s}$ is nonlinear with a maximum at the spacer length $\mathrm{s}=6$. A similar trend in the $\mathrm{cmc}$ dependence on the spacer length was found for gemini surfactants with dodecyl chains and polymethylene spacer, alkanediyl- $\alpha, \omega$-bis(dimethyldodecylammonium bromides) referred to as 12-s-12 [39]. The existence of the cmc maximum is attributed to the conformational changes of the spacer. The preferential cis conformation of alkyl chains is assumed for gemini molecules with the short spacer values ( $s=2$ to 6 ) which may result in some contacts between the two alkyl chains which results in slight increase of the Gibbs energy and cmc. Above s $=6$, the slight $\mathrm{cmc}$ decrease corresponds to the reported decrease of $\mathrm{cmc}$ for single-chain surfactants $\mathrm{C}_{12} \mathrm{H}_{25}\left(\mathrm{C}_{\mathrm{s} / 2}, / \mathrm{H}_{\mathrm{s}+1}\right) \mathrm{N}^{+}\left(\mathrm{CH}_{3}\right)_{2} \mathrm{Br}^{-}$which have a side chain length equal to that of gemini molecules spacer. The cmc decrease was found for the side chain length $\mathrm{s} / 2>3$ which corresponds to the spacer length of gemini surfactants above $6 \mathrm{CH}_{2}$ groups [57]. As results from Fig. 3, the cmc curve of $\mathbf{u}(\mathbf{s})$ lies below that of the gemini surfactants with dodecyl chains and a polymethylene spacer $12-\mathrm{s}-12$. This is expectable due to the fact that the investigated gemini molecules with an urea group in each of the alkyl chains (total number of atoms in the chain 16) have the alkyl chains longer than a dodecyl chain and consequently, their cmc values are smaller than those of 12-s-12 for all inspected spacer values. On the other hand, the dependence of area per surfactant molecule of $\mathbf{u}(\mathbf{s})$ on the spacer length shows the increasing trend with the increasing number of $\mathrm{CH}_{2}$ groups in the spacer and roughly fits the area values of 12-s-12 gemini surfactants (Fig 4). 


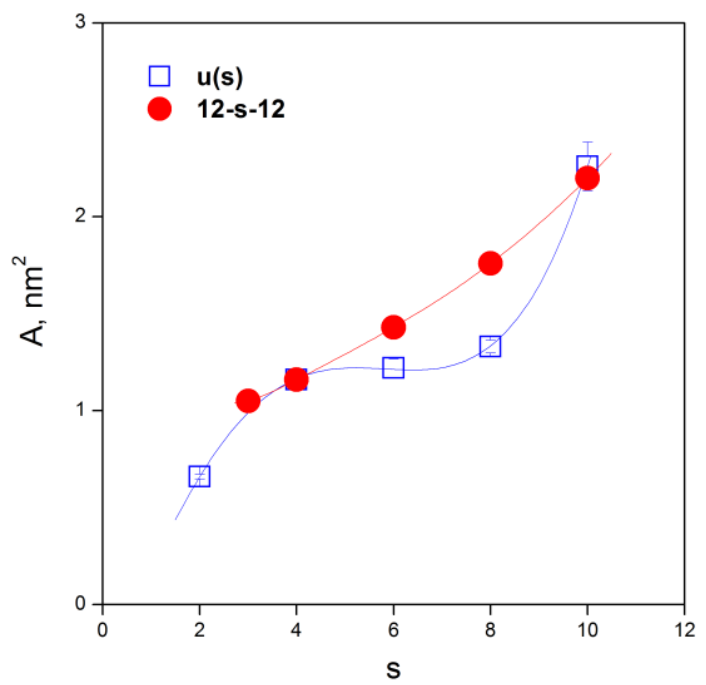

Fig. 4. Area per gemini molecule $A$ at the air/water interface plotted as a function of $\mathbf{u}(\mathbf{s})$ gemini surfactant spacer length s. A of 12-s-12 gemini surfactants [22] is indicated by solid circles.

This may indicate the role of structure and geometry of the head part of a gemini molecule (two ammonium heads and the spacer) which predominantly affects its interfacial area value. Generally, the monotonous increase of area per surfactant molecule for gemini molecules with the increasing polymethylene spacer length in the region of s values ranging from 2 to $10-12$ is attributed to the rigidity of a polymethylene spacer of medium length [40] which results in the increased separation of polar parts in a gemini molecule. According to [46], there are two characteristic distances in a gemini micelle. A structural distance $d_{s}$ which corresponds to the extended length of the spacer and is equal to $d_{s}=$ $0.1265(\mathrm{~s}+1)$ in nanometres [58] given that one-half the bond length is assumed at both ends of the spacer. The second distance is a thermodynamic equilibrium distance $\mathrm{d}_{\mathrm{T}}$ characterizing the displacement of surfactant molecules at the interface which is about 0.7 $-0.9 \mathrm{~nm}$ [46]. In the previous study [41] we have found that the interfacial area of gemini molecule with a short spacer (12-2-12) may be related only to its steric arrangement and is independent of hydrophobic interactions at the interface. As a consequence, the interfacial area value for gemini molecules with $s=2$ is determined only by the structural distance $d_{s}$. The role of the spacer steric arrangement as a crucial factor controlling the interfacial area value in case of short spacers, corresponds to the found values of interfacial area for $\mathbf{u} \mathbf{2}, \mathbf{u} \mathbf{4}$ and 12-2-12, 12-4-12, respectively, which overlap and show no significant difference between each other (Fig. 4). As shown in Fig. 3, the interfacial values start to differ from each other at the medium spacer lengths 6 and $8 \mathrm{CH}_{2}$ groups. At $\mathrm{s}=6,8$ the interfacial area values for gemini molecules with urea groups in the alkyl chains are smaller than those of 
12-6-12 and 12-8-12 gemini surfactants (Fig. 3). For the medium spacer lengths of gemini surfactants, $d_{s}$ approaches $d_{T}$ [46] which indicates an increasing influence of the thermodynamic equilibrium distance $\mathrm{d}_{\mathrm{T}}$ on the arrangement of gemini molecules at the interface. At the medium spacer lengths $(s=6,8)$, the arrangement of molecules and the interfacial area are controlled not only by the structural distance $\mathrm{d}_{\mathrm{s}}$ which is exclusively a function of the spacer length but also by $\mathrm{d}_{\mathrm{T}}$ which includes a contribution from the intermolecular interactions (interactions between polar head groups and hydrophobic interactions between alkyl chains). It may be assumed that the slight decrease in the interfacial area values of gemini molecules $\mathbf{u 6}$ and $\mathbf{u 8}$ compared to those of 12-6-12 and 12-8-12 (Fig. 4), is attributed to the presence of urea groups in the alkyl tails. A hydrogen bonding is present between two urea groups with the hydrogen bonding distance $0.218 \mathrm{~nm}$, as results from quantum mechanical calculations [16]. The effect of the intermolecular hydrogen bonding in urea-based gemini surfactants $\mathbf{u}(\mathbf{s})$ may be more pronounced at the medium spacer lengths when the rigid spacer is long enough to allow intermolecular hydrophobic interactions between alkyl chains of neighbouring gemini molecules including the mutual approach of neighbouring $\mathrm{NH}-\mathrm{C}=\mathrm{O}-\mathrm{NH}$ groups to the distance stated above which is necessary for the formation of hydrogen bond. The contribution of hydrogen bonding interactions within hydrophobic interactions of alkyl chains may be responsible for the slight interfacial area decrease of $\mathbf{u 6}$ and $\mathbf{u 8}$, as it is shown in the schematic representation of gemini molecules arrangement at the air/water interface (Fig.5) with the indication of the intermolecular hydrogen bonding between neighbouring urea groups. As an example shown in Fig 5, the dotted lines represent a symmetrical triatomic hydrogen bonding between the oxygen of carbonyl group and both hydrogens of the neighbouring molecule amino groups.

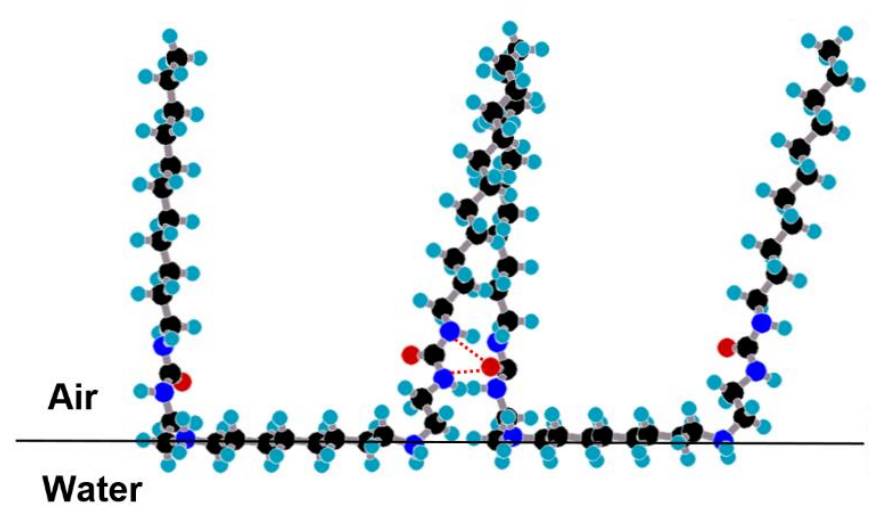

Fig. 5. Schematic representation of the arrangement of $\mathbf{u} 8$ gemini molecules at the air/water interface. 
For gemini surfactants with a longer spacer $(s=10)$, the interfacial area values of $\mathbf{u 1 0}$ and 12-10-12 overlap again. This indicates that another factor controlling the interfacial area value sets on. From $s=10$, the increasing spacer flexibility starts to predominate among the interactions influencing the interfacial area value of gemini molecule. At $s \geq 10$, the spacer becomes too hydrophobic to remain in contact with hydrophilic phase and moves to the air side of the interface adopting a bent conformation [40]. The effect of a flexible spacer tends to move the head groups closer to each other. This factor may be dominant as compared to other influences (hydrophobic interaction of alkyl tails, hydrogen bonding between urea groups) which results in almost identical values of area per surfactant molecule for gemini surfactants with longer spacer such as $\mathbf{u 1 0}$ and 12-10-12 (Fig. 3).

\subsection{Hydrodynamic size of micelles}

In Fig. 6 and 7, dependences of micelle hydrodynamic diameter d vs. surfactant concentration $\mathrm{c} / \mathrm{cmc}$ for all investigated gemini surfactants $\mathbf{u}(\mathbf{s})$ calculated from the dynamic light scattering results, are shown.

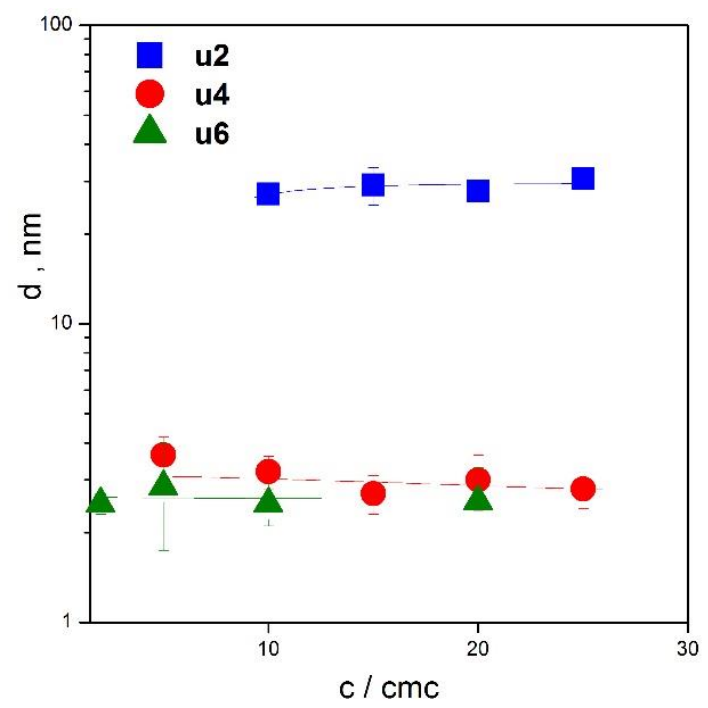

Fig. 6. Hydrodynamic size of gemini micelles u2, u4, u6 plotted as a function of surfactant concentration. 


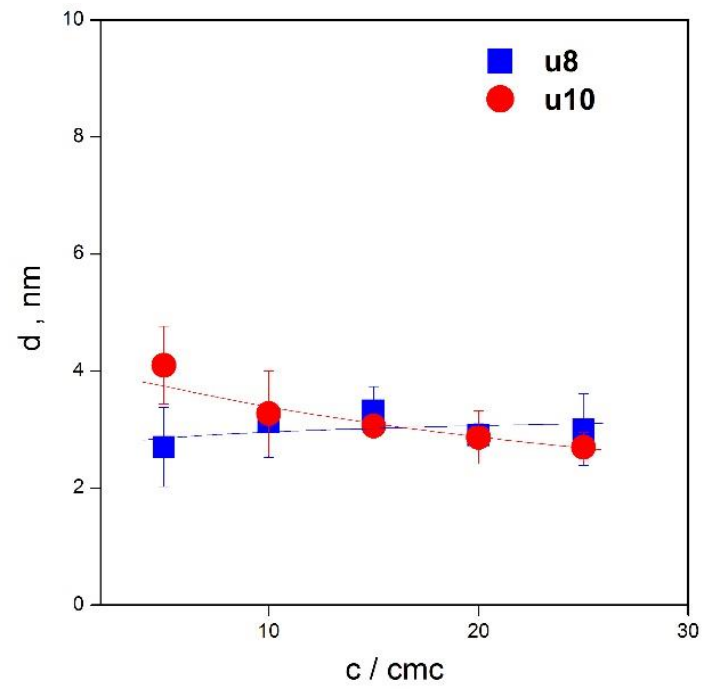

Fig. 7. Hydrodynamic size of gemini micelles $\mathbf{u 8}, \mathbf{u 1 0}$ plotted as a function of surfactant concentration.

The dependence of hydrodynamic diameter on $\mathrm{c} / \mathrm{cmc}$ is linear and almost does not change with the surfactant concentration (Figs. 6 and 7). A substantially larger particle size with the values above $20 \mathrm{~nm}$ was found for $\mathbf{u} 2$ (Fig. 6). This indicates a stronger aggregation tendency of $\mathbf{u} \mathbf{2}$ molecules which corresponds to the area per surfactant molecule values (Fig. 4) where the role of the spacer steric arrangement turned out to be a main factor controlling the micellar morphology. A significant increase in surfactant prolate major axis for 10-2-10 and 10-3-10 (gemini surfactants with decyl chains) was found with the increasing surfactant weight percentage, as results from small-angle neutron scattering measurements [42]. This size increase is no more observable for longer spacers $s=4$ to 12 $[42,43]$. Similarly, no significant increase in size was observed for micelles composed of gemini molecules with dodecyl chains 12 -s-12 and the spacer values $\mathrm{s}=4,6,8,10,12$ [59]. As results from the dependence of the hydrodynamic micelle diameter on the spacer number at the constant gemini surfactant concentration $10 \mathrm{x} \mathrm{cmc} \mathrm{(Fig.} \mathrm{8),} \mathrm{an}$ approximately constant micelle size was found for $\mathbf{u}(\mathbf{s})$ micelles with the medium spacer length (u4 to u10), which corresponds to the data from the references stated above. A weak minimum in the micelle diameter vs. spacer number dependence was found for $\mathbf{u} 6$ micelles, however, not statistically significant. The small diameter values for $\mathbf{u} 4$ to $\mathbf{u 1 0}$ micelles being in the range $2-4 \mathrm{~nm}$ indicate a region of the formation of stable spherical micelles. For $\mathbf{u} 2$ gemini surfactant, larger aggregates with the size between $27-30 \mathrm{~nm}$ are observed (Fig. 8). 


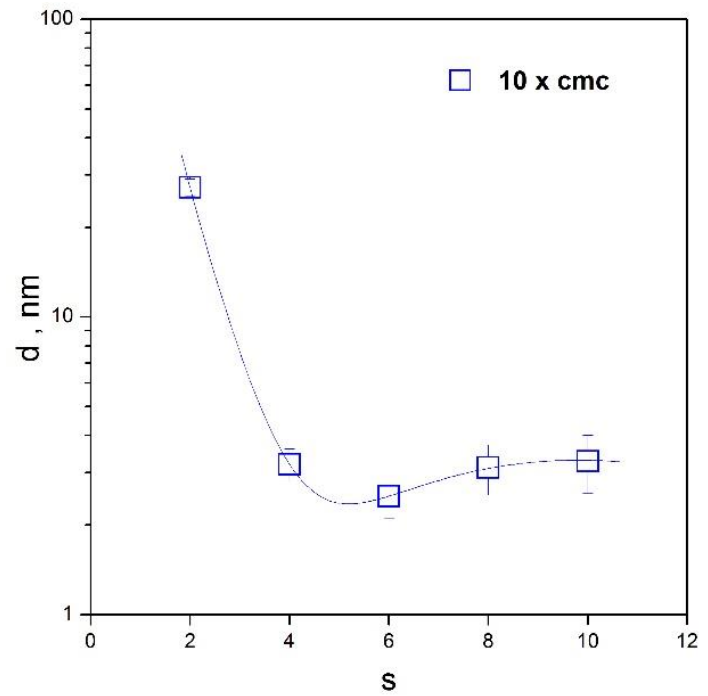

Fig. 8. Hydrodynamic size of $\mathbf{u}(\mathbf{s})$ gemini micelles plotted as a function of surfactant spacer length $\mathrm{s}$ at the surfactant concentration $10 \mathrm{x} \mathrm{cmc}$.

\subsection{Zeta potential of micelles}

Concentration dependences of zeta potential for micelles of $\mathbf{u}(\mathbf{s})$ gemini surfactants are shown in Fig. 9 for all spacer lengths. For u2, u4 and u6 micelles, the dependences show a moderate zeta potential increase at the low surfactant concentration.

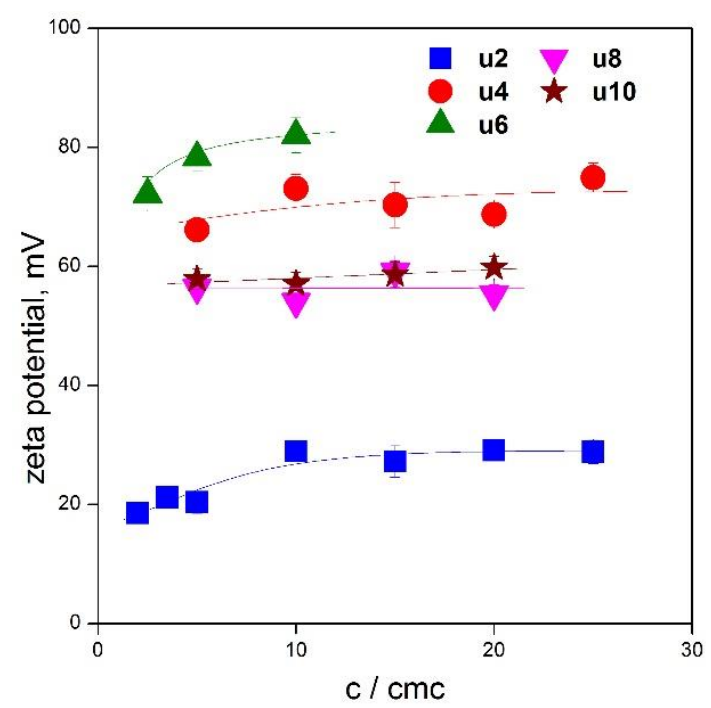

Fig. 9. Zeta potential of $\mathbf{u}(\mathbf{s})$ gemini micelles plotted as a function of surfactant concentration.

At higher c/cmc values, zeta potential levels off to become constant. For $\mathbf{u} 8$ and $\mathbf{u 1 0}$, the dependences are approximately linear with the slope values close to zero. Zeta potential of single chain quaternary ammonium surfactant dodecyltrimethylammonium bromide 
(DTAB) was found to be near to $60 \mathrm{mV}$ in DTAB aqueous solution at the concentration close to the cmc [60]. DTAB forms only spherical micelles even at high concentrations [61]. The DTAB zeta potential value at its $\mathrm{cmc}$ is close to the zeta potential values of $\mathbf{u} 8$ and $\mathbf{u 1 0}$ indicating the spherical shape of gemini molecules with the spacer length less than 12 carbon atoms. The indication of micellar growth of gemini surfactants with the spacer length $4 \mathrm{CH}_{2}$ groups and the alkyl chain length larger than 12 carbon atoms was confirmed by the changes in zeta potential values [60]. Plotting the zeta potential values against the spacer number at the constant surfactant concentration $5 \mathrm{x} \mathrm{cmc} \mathrm{(Fig.} \mathrm{10)} \mathrm{gives} \mathrm{a} \mathrm{non-linear}$ dependence of zeta potential on the spacer length which shows the maximum zeta potential at the medium spacer length $s=6$. The formation of stable monodisperse spherical micelles at this spacer length results in large surface charge of micelles and larger positive zeta potential values. As the spacer length decreases towards short spacer $s=2$, the decreasing surface charge and zeta potential indicate a denser arrangement of polar parts of gemini molecules in the micelle surface layer and the resulting micellar growth which was confirmed from the light scattering results.

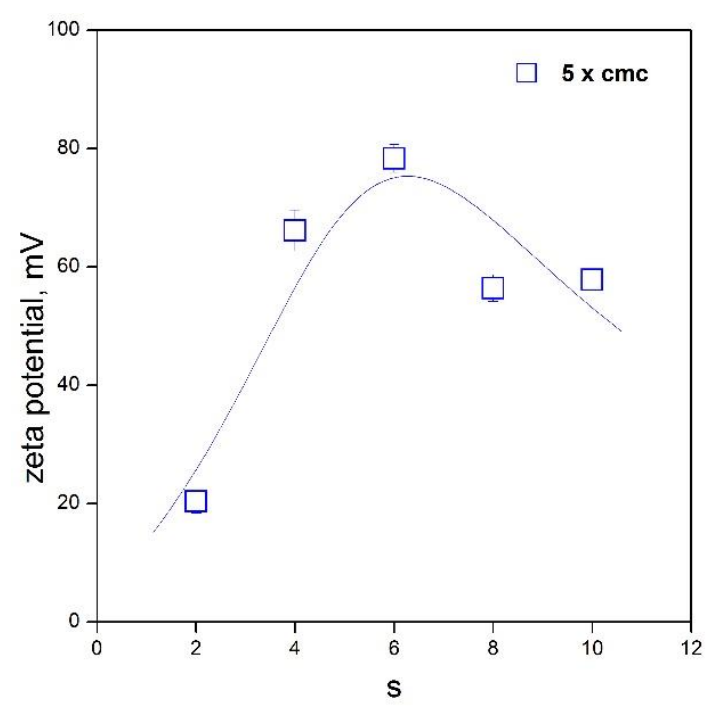

Fig. 10. Zeta potential of $\mathbf{u}(\mathbf{s})$ gemini micelles plotted as a function of surfactant spacer length $s$ at the surfactant concentration $5 \mathrm{x}$ cmc.

\subsection{Micelle aggregation number}

The dependence of micelle aggregation number $\mathrm{N}$ on surfactant concentration c/cmc is plotted in Fig. 11 for all spacer length values of $\mathbf{u ( s )}$ gemini surfactants. 


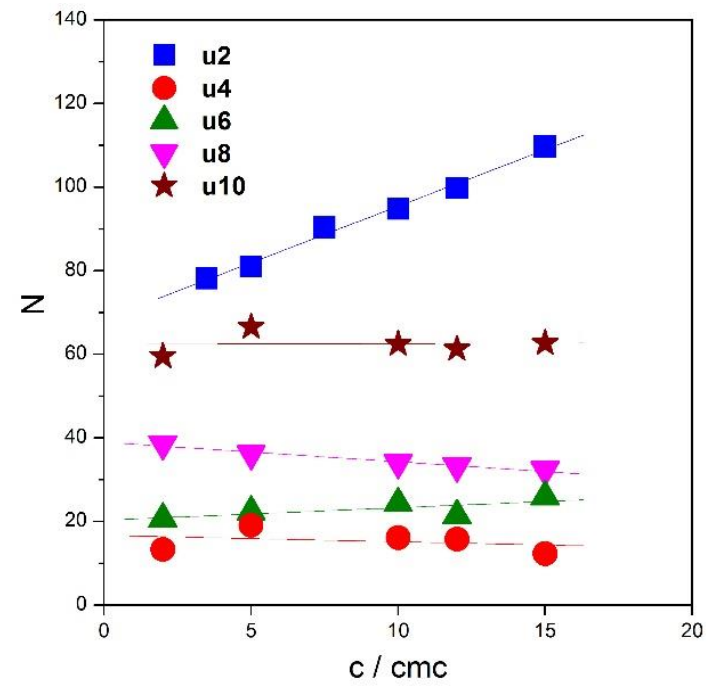

Fig. 11. Micelle aggregation number of $\mathbf{u}(\mathbf{s})$ gemini surfactants plotted as a function of surfactant concentration.

As follows from the plot, the aggregation number of gemini surfactants does not change much with surfactant concentration for all spacer lengths within the investigated concentration range except for $\mathrm{s}=2$. $\mathbf{u} 2$ micelles show an increased aggregation behaviour which results in the micellar growth. This finding corresponds with the low interfacial area for $\mathbf{u} 2$ gemini molecules (Fig. 4) which is also an indication of the increased aggregation tendency of $\mathbf{u} 2$. The plot of the slopes in the $\mathrm{N}$ vs. c/cmc dependence shows a significant difference between $\mathbf{u} 2$ micelles and micelles of gemini molecules with other spacer values (Fig. 12).

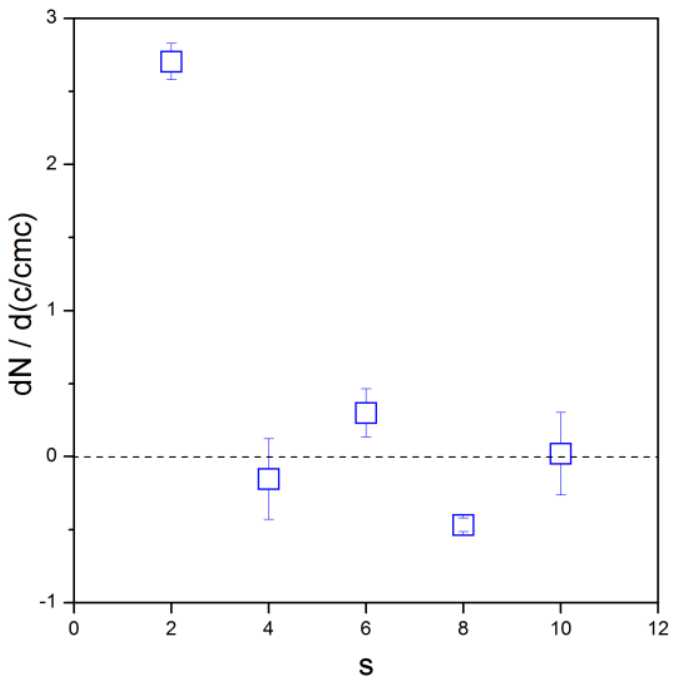

Fig. 12. Slope values of concentration dependences of the $\mathbf{u}(\mathbf{s})$ micelle aggregation number plotted as a function of surfactant spacer length s. 
The slope value for $\mathbf{u} 2$ micelles lies between 2 and 3 whereas the slope values for other spacer lengths are scattered around zero. A similar difference in the slopes of concentration dependences of micelle aggregation number was observed for the gemini surfactant with the short methylene spacer 12-3-12 on the one hand and for micelles composed of gemini molecules with longer spacer values s $>4$ on the other [28]. The corresponding single chain quaternary ammonium surfactant DTAB shows only a very small increase in N [38] with the increasing concentration. Therefore, the tendency to micellar growth is primarily determined by the structural arrangement of gemini molecules with the short spacer $(s=2$, 3) which results in the dense packing of molecules at the interface and by a significant aggregation of gemini molecules in the volume. This structural factor disappears with the increasing spacer length, as the structural distance $d_{s}$ approaches the thermodynamic distance $d_{T}$ which is discussed in the Surface tension section. The aggregation properties of gemini molecules with the spacers of medium length show the aggregation behaviour similar to that of a single chain analogue DTAB which forms stable spherical micelles with the constant hydrodynamic size and aggregation number relatively independent of surfactant concentration. The plot $\mathrm{N}$ vs. gemini surfactant concentration (Fig. 11) can be rearranged in a plot of micelle aggregation number against the spacer number at all investigated surfactant concentrations. It is obvious from the plot that the concentration dependence of $\mathrm{N}$ appears only for $\mathbf{u} \mathbf{2}$ gemini surfactant. The results indicate a large number values for $\mathbf{u} 2$ being in the range 80-110 (Fig. 13).

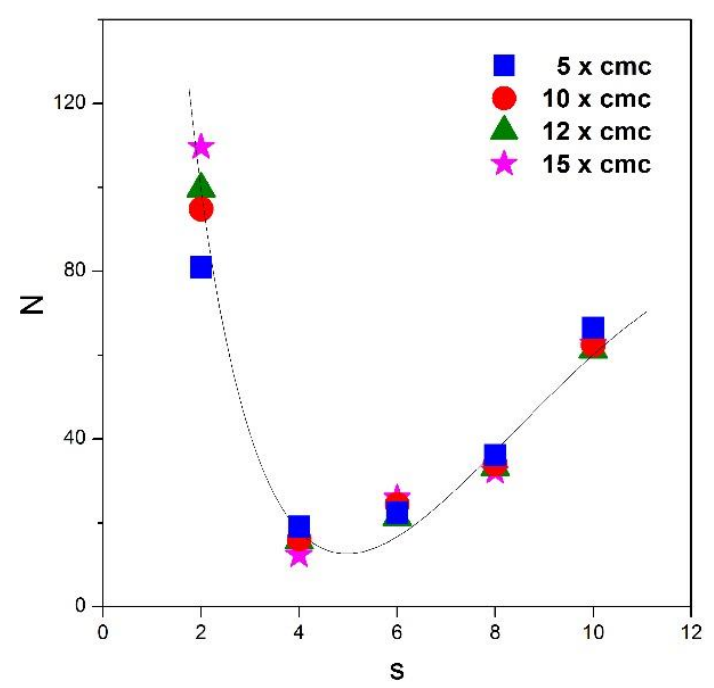

Fig. 13. Micelle aggregation number of $\mathbf{u}(\mathbf{s})$ gemini surfactants plotted as a function of surfactant spacer length s for all investigated concentrations. 
The $\mathbf{u} 4$ - u10 gemini surfactant micelles show lower aggregation numbers between 16 63. As results from the plot, a moderate increase in $\mathrm{N}$ with the increasing spacer number $s$ is observed. The moderate increase of aggregation number with the surfactant concentration showing the interval of $\mathrm{N}$ values 25 - 55 was found for gemini surfactants with polymethylene spacer s > 5, 12-6-12, 12-8-12, 12-10-12 [28]. Our dynamic light scattering results do not show significant changes in micellar size with the spacer number $\mathbf{s}$ (Fig. 6,7) and the micelles are assumed to remain spherical or spheroidal. Physical characteristics of $\mathbf{u}(\mathbf{s})$ micelles obtained by the techniques mentioned above are shown in Table 1. 
Table 1. Micellar characteristics of urea-based surfactants. cmc - critical micelle concentration, $\mathrm{A}$ - area per surfactant molecule, $\mathrm{d}-$ micelle hydrodynamic diameter, $\zeta$ zeta potential, $\mathrm{N}$ - aggregation number. $\mathrm{d}, \zeta$ and $\mathrm{N}$ were determined at the gemini surfactant concentration $10 \mathrm{x} \mathrm{cmc}$.

\begin{tabular}{cccccc}
\hline Compound & $\begin{array}{c}\mathbf{c m c} \\
\mathbf{1 0}^{-4} \mathbf{m o l} / \mathbf{L}\end{array}$ & $\begin{array}{c}\mathbf{A} \\
\mathbf{n m}^{\mathbf{2}}\end{array}$ & $\begin{array}{c}\mathbf{d} \\
\mathbf{n m}\end{array}$ & $\begin{array}{c}\boldsymbol{\zeta} \\
\mathbf{m V}\end{array}$ & $\mathbf{N}$ \\
\hline $\mathbf{u 2}$ & $0.54 \pm 0.01$ & $0.66 \pm 0.01$ & $27.2 \pm 1.8$ & $20.4 \pm 2.0$ & 94.8 \\
$\mathbf{u 4}$ & $2.02 \pm 0.01$ & $1.16 \pm 0.02$ & $3.2 \pm 0.4$ & $66.2 \pm 3.5$ & 16.1 \\
$\mathbf{u 6}$ & $3.19 \pm 0.02$ & $1.22 \pm 0.05$ & $2.5 \pm 0.4$ & $78.3 \pm 2.4$ & 24.3 \\
$\mathbf{u 8}$ & $2.02 \pm 0.02$ & $1.33 \pm 0.03$ & $3.1 \pm 0.6$ & $56.4 \pm 2.3$ & 34.0 \\
$\mathbf{u 1 0}$ & $1.32 \pm 0.03$ & $2.26 \pm 0.13$ & $3.3 \pm 0.7$ & $57.8 \pm 1.8$ & 62.5 \\
\hline
\end{tabular}

\subsection{Small angle neutron scattering}

Micelles of three urea-based gemini surfactants with a short (u2) medium (u6) and long (u10) spacer were examined by small-angle neutron scattering technique. The scattering data for the three selected surfactants at the three concentrations well above cmc are shown in Fig 14. All scattering curves show the characteristic micelle interference peak, which is a consequence of a strong electrostatic repulsion between the charged micelles. The $\mathbf{u} 2$ solutions, in addition, show a strong increase towards small angles, which can be attributed to aggregates of a small non-dissolved fraction of the surfactant molecules. In the fitting procedure, this extra scattering had been taken into account as an additional background caused by large aggregates. 

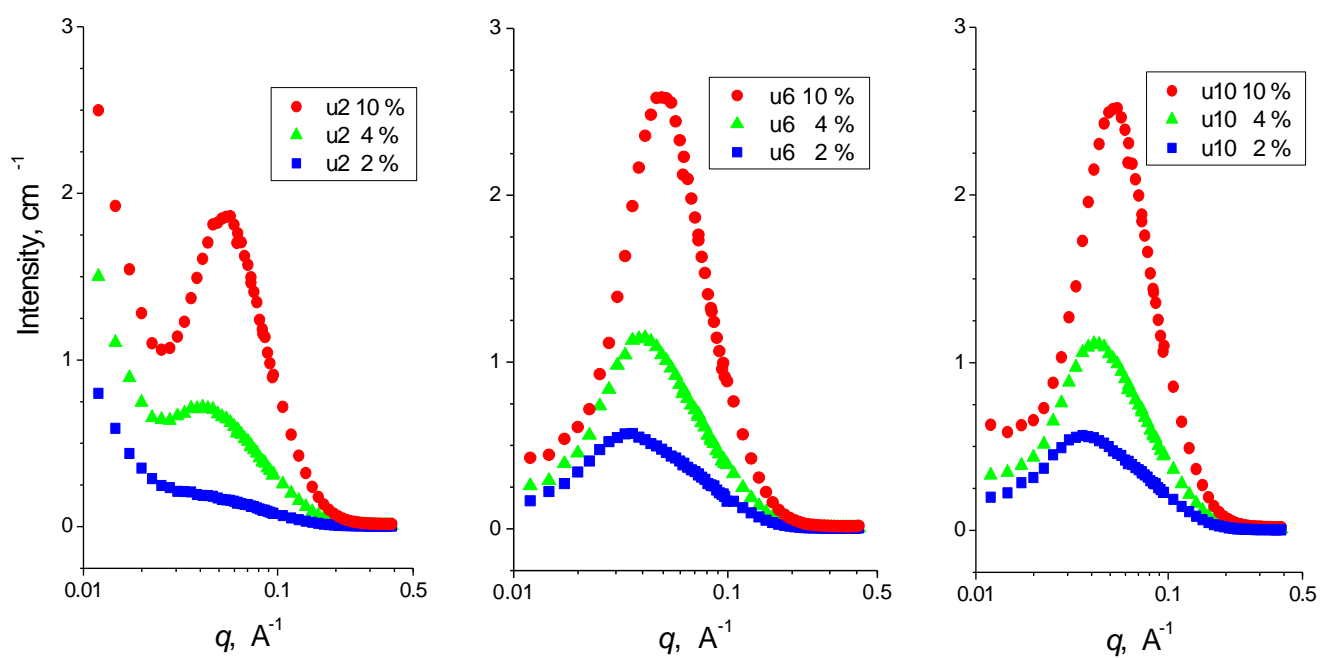

Fig. 14. SANS scattering curves for micellar solutions of $\mathbf{u 2}, \mathbf{u} \mathbf{6}$ and $\mathbf{u 1 0}$ surfactants at concentrations of 2,4 and $10 \mathrm{wt} \%$ in heavy water.

The micelle aggregation numbers have been determined by considering the volume of the ellipsoidal micelles and the molar volume of the surfactants. A brief comparison of aggregation number values obtained from time resolved fluorescence quenching (TRFQ) and neutron scattering (SANS) methods is provided in Fig. 15. 


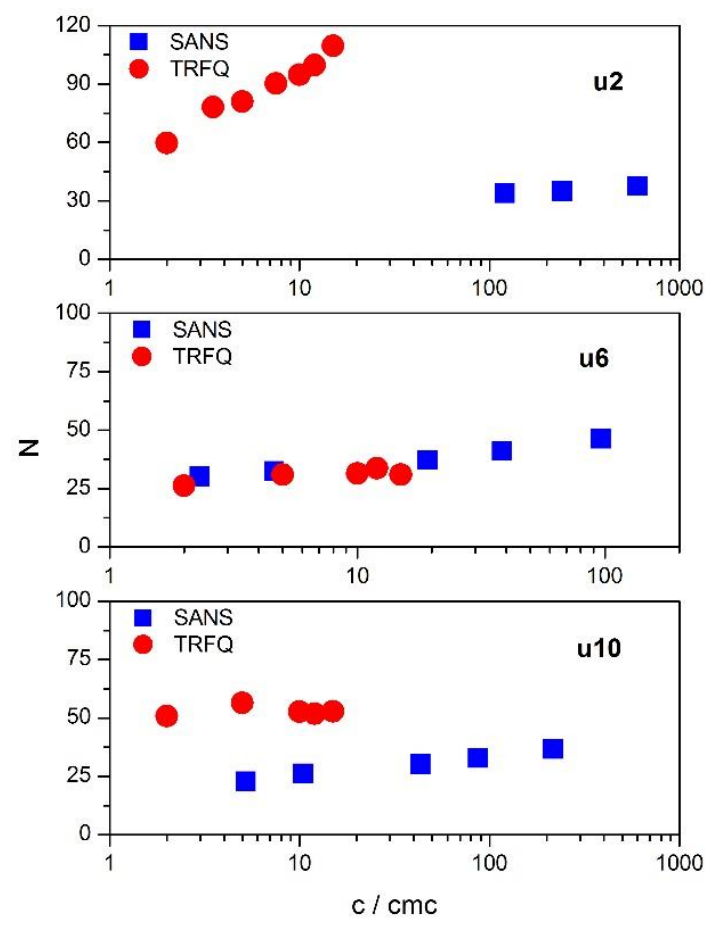

Fig. 15. Comparison of aggregation number of $\mathbf{u} 2, \mathbf{u} 6$ and $\mathbf{u 1 0}$ micelles calculated from small angle neutron scattering (SANS) and time-resolved fluorescence quenching experiments (TRFQ).

As results from the figure, the best match of micelle aggregation number was reached for the region of urea-based gemini molecules with the medium spacer length (u6) which form stable micelles uniform in size. Towards shorter and longer spacers, the difference in micelle aggregation number slightly increases. Larger values are obtained from the fluorescence method which is most noticeable for the gemini surfactant with the shortest spacer $\mathbf{u} 2$. This difference may be attributed to the increased size polydispersity of aggregates and possible solution inhomogeneities resulting from the observed strong aggregation of $\mathbf{u} \mathbf{2}$ at high concentration which was required for the SANS measurements.

\subsection{Antimicrobial activity}

Antimicrobial activity of urea-based surfactants $\mathbf{u ( s )}$ was evaluated against grampositive bacteria, gram-negative bacteria and fungi by determining the minimum bactericidal concentration (MBC) utilizing the diffusion agar technique. The antimicrobial efficiency of urea-based surfactants is expressed as the logarithm of inverse MBC values which are plotted in Fig. 16 with the last two points indicating the antimicrobial activity of the reference compounds cetylpyridinium bromide (CPyB) benzyldodecyldimethylammonium bromide (BDDAB). For the sake of comparision with other widely used 
quaternary ammonium disinfectants with more gemini-like molecular structure, the published data for a double-chain surfactant with a single head group didecyldimethylammonium chloride, which is widely used in formulations for the disinfection and antisepsis both in the medical area as well as in the food industry, show the values of $\log (1 / \mathrm{MBC}, \mathrm{L} / \mathrm{mol})$ equal to 5.1 for Staphylococcus aureus and 3.8 and smaller for gram-negative strains, respectively [62]. As results from Fig. 16, bactericidal activity of $\mathbf{u}(\mathbf{s})$ gemini surfactants is better than that of the stated reference disinfectants for all spacer lengths except for $\mathrm{s}=2$.

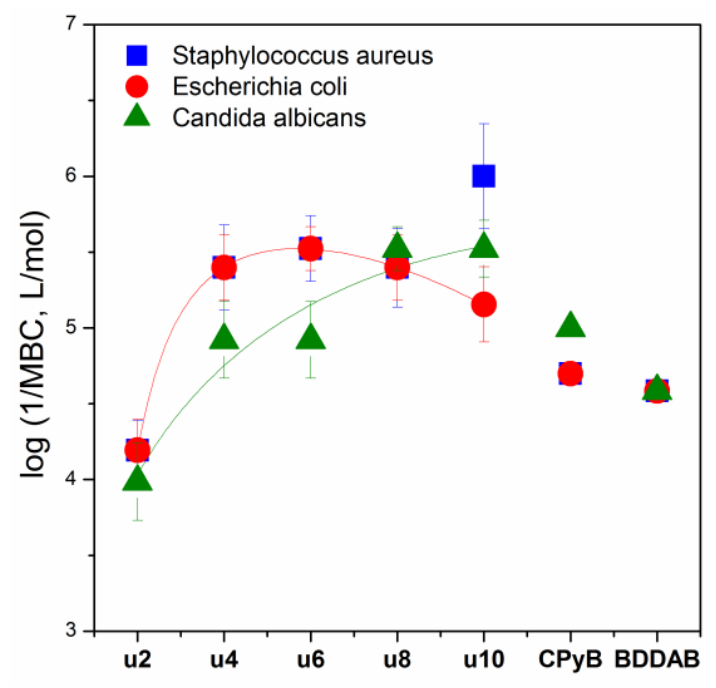

Fig. 16. Plot of the logarithm of inverse minimum bactericidal concentration of $\mathbf{u}(\mathbf{s})$ gemini surfactants plotted as a function of surfactant spacer length s. The last two points in the plot are the reference compounds.

As results from Fig. 16, u(s) molecules exhibit the antimicrobial activity expressed in log $1 / \mathrm{MBC}$ values which is found to be larger than that of the reference compounds $\mathrm{CPyB}$ and $\mathrm{BDDAB}$ for all investigated spacer lengths except for $\mathrm{s}=2$. A comparison of antimicrobial activity of urea-based gemini surfactants with bisammonium gemini surfactants with polymethylene spacer containing no urea groups in the molecular structure does not indicate a significant influence of the presence urea groups in the molecular structure of gemini surfactants on the change in their bactericidal activity (see the supplementary material for details). The interaction of cationic surfactants with biomembrane through the adsorption of surface-active molecules on the outer cellular membrane of microorganisms is the most preferable explanation of the antimicrobial activity mechanism at the molecular level [63]. The positively charged headgroups of cationic surface-active molecules interact 
with a negatively charged bacterial cell membrane. As a result, a membrane disruption occurs with the aid of electrostatic and hydrophobic interactions followed by a release of cytoplasmic constituents and subsequent cell death. The data shown in Fig. 16 indicate a maximum in the dependence of antimicrobial activity on surfactant spacer number s. This maximum was found at the spacer number $\mathrm{s}=6(\mathbf{u 6})$ and is identical for both grampositive (Staphylococcus aureus) and gram-negative (Escherichia coli) bacteria. A similar increase in antimicrobial activity was observed for amido-amine-based cationic gemini surfactants when the gemini molecule spacer length was increased from $3 \mathrm{CH}_{2}$ groups to 6 $\mathrm{CH}_{2}$ groups [64]. The maximum in antimicrobial activity for fungi (Candida albicans) seems to be reached at a higher spacer length of $10 \mathrm{CH}_{2}$ groups (antimicrobial activity of gemini molecules with longer spacer than $\mathrm{s}=10$ was not investigated). This may be related to a different composition of cellular membrane of bacteria and fungi which predetermines the level of interaction of $\mathbf{u}(\mathbf{s})$ molecules with microorganisms. Two layers can be distinguished in the Candida albicans cell wall. The outer layer is highly enriched with Oand N-linked mannose polymers whereas the inner layer contains the skeletal polysaccharides chitin and $\beta$-1,3-glucan, which confer strength and cell shape [65]. A similar cell membrane structure consisting of inner and outer membrane can be observed also for the gram-negative Escherichia coli but its structure is not so complex. The inner membrane is a symmetric lipid bilayer composed of phospholipids and integral membrane proteins. The outer membrane is an asymmetric lipid bilayer containing phospholipids in its inner leaflet and lipopolysaccharide in its outer leaflet [66]. The complex, polymer-like structure of Candida albicans cell membrane may hinder easy hydrophobic and electrostatic interaction with gemini molecules. To reach the antimicrobial efficiency of $\mathbf{u}(\mathbf{s})$ surfactants comparable with that against gram-negative Escherichia coli, a stronger hydrophobic interaction is required to overcome the complex cell wall structure of Candida albicans. This results in the requirement for a more hydrophobic gemini molecule for the interaction with Candida albicans cells. As a result, the maximum in antimicrobial efficiency is shifted towards gemini surfactants with longer spacer length of $10 \mathrm{CH}_{2}$ groups (Fig. 16). 


\section{Conclusions}

The present study provides an information about the synthesis and physicochemical, aggregation and biological properties of molecules of a series of novel cationic urea-based gemini surfactants with a variable spacer length. The results indicate a stronger aggregation tendency of urea-based surfactants with a short length of polymethylene spacer ( $2 \mathrm{CH}_{2}$ groups) which was confirmed by all applied experimental methods (neutron and light scattering, surface tension, fluorescence, zeta potential measurements). As results from the comparison with the gemini surfactants without urea groups in the alkyl tails and polymethylene spacer, the presence of urea groups even enhances the aggregation properties of gemini molecules through additional hydrogen bonding interactions. At the medium spacer lengths up to $10 \mathrm{CH}_{2}$ groups, all applied methods indicate the presence of monodisperse spherical micelles of a relatively constant size and aggregation number. This is to relate to the diminished influence of hydrogen bonding between urea groups on the aggregation of gemini molecules with the increased spacer length. The antimicrobial activity shows nonlinear parabolic behaviour vs. spacer length with the maximum antimicrobial activity found at the spacer length of $6 \mathrm{CH}_{2}$ groups for bacteria and $8 \mathrm{CH}_{2}$ groups for fungi. This is to attribute to a different composition of cell membrane of the used microorganisms. The current research results provide a good basis for the development of new aggregation modifiers due to the ability of these molecules to control aggregation behaviour (vesicles formation, gelation) as a result of their strong hydrogen bonding.

\section{Acknowledgements}

This work was supported by the Slovak Research and Development Agency Grant No. APVV-0516-12 Small Molecules in Biomedical Research. 


\section{References}

[1] A.K. Hüsecken, F. Evers, C. Czeslik, M. Tolan, Effect of Urea and Glycerol on the Adsorption of Ribonuclease A at the Air-Water Interface, Langmuir. 26 (2010) 13429-13435. doi:10.1021/la102222z.

[2] Z. Yang, P. Xiu, B. Shi, L. Hua, R. Zhou, Coherent Microscopic Picture for UreaInduced Denaturation of Proteins, J. Phys. Chem. B. 116 (2012) 8856-8862. doi:10.1021/jp304114h.

[3] D. Wells, C.J. Drummond, Nonionic n-Hexyl, n-Heptyl, and n-Octyl Urea Surfactants: Some Physicochemical Properties, Langmuir. 15 (1999) 4713-4721. doi:10.1021/la9815421.

[4] U. Thapa, K. Ismail, Urea effect on aggregation and adsorption of sodium dioctylsulfosuccinate in water, J. Colloid Interface Sci. 406 (2013) 172-177. doi:10.1016/j.jcis.2013.06.009.

[5] S. Kumar, Z.A. Khan, N. Parveen, Kabir-ud-Din, Influence of different ureas on aggregational properties of aqueous surfactant systems, Colloids Surf. Physicochem. Eng. Asp. 268 (2005) 45-51. doi:10.1016/j.colsurfa.2005.05.022.

[6] S. Kumar, N. Parveen, Kabir-ud-Din, Effect of Urea Addition on Micellization and the Related Phenomena, J. Phys. Chem. B. 108 (2004) 9588-9592. doi:10.1021/jp036552w.

[7] P.K.D. Gupta, S.P. Moulik, Effects of urea and a nonionic surfactant on the micellization and counterion binding properties of cetyltrimethyl ammonium bromide and sodium dodecyl sulfate, Colloid Polym. Sci. 267 (1989) 246-254. doi:10.1007/BF01410582.

[8] J. Hao, T. Wang, S. Shi, R. Lu, H. Wang, Electron Spin Resonance Study of Effect of Urea on Microenvironmental Properties of Alkylbenzenesulfonate Micellar Solutions, Langmuir. 13 (1997) 1897-1900. doi:10.1021/la9604442.

[9] R.K. Mahajan, R. Kaur, V.K. Aswal, Effect of urea on the aggregation behavior of gemini surfactants and their mixed micelles with Pluronic L64, Colloids Surf. Physicochem. Eng. Asp. 419 (2013) 61-68. doi:10.1016/j.colsurfa.2012.11.049.

[10] U. Anand, S. Mukherjee, Microheterogeneity and Microviscosity of F127 Micelle: The Counter Effects of Urea and Temperature, Langmuir. 30 (2014) 1012-1021. doi:10.1021/la4049192.

[11] J. Broecker, S. Keller, Impact of Urea on Detergent Micelle Properties, Langmuir. 29 (2013) 8502-8510. doi:10.1021/la4013747.

[12] M.A. Jouani, S. Szönyi, H. Trabelsi, S.Y. Dieng, A. Cambon, H.J. Watzke, Synthesis and aggregation properties of new fluorine-containing double-chain amphiphiles derived from di- and tri-substituted ureas, Supramol. Sci. 2 (1995) 117-123. doi:10.1016/0968-5677(96)89076-6.

[13] A. Raghavanpillai, S. Reinartz, K.W. Hutchenson, Hydrophobic and oleophobic surface modification using fluorinated bis-urea and bis-amide gelators, J. Fluor. Chem. 130 (2009) 410-417. doi:10.1016/j.jfluchem.2009.01.006.

[14] C.M.C. Faustino, A.R.T. Calado, L. Garcia-Rio, New Urea-Based Surfactants Derived from $\alpha, \omega$-Amino Acids, J. Phys. Chem. B. 113 (2009) 977-982. doi:10.1021/jp807396k.

[15] C.M.C. Faustino, A.R.T. Calado, L. Garcia-Rio, Dimeric and monomeric surfactants derived from sulfur-containing amino acids, J. Colloid Interface Sci. 351 (2010) 472477. doi:10.1016/j.jcis.2010.08.007.

[16] E. Yilgör, E. Burgaz, E. Yurtsever, İ. Yilgör, Comparison of hydrogen bonding in polydimethylsiloxane and polyether based urethane and urea copolymers, Polymer. 41 (2000) 849-857. doi:10.1016/S0032-3861(99)00245-1. 
[17] F.H. Florenzano, L.G.C. dos Santos, I.M. Cuccovia, M.V. Scarpa, H. Chaimovich, M.J. Politi, Urea-Induced Decrease of Anion Selectivity in Surfactant Aggregates, Langmuir. 12 (1996) 1166-1171. doi:10.1021/la9505834.

[18] J. Almeida, Dicationic Alkylammonium Bromide Gemini Surfactants. Membrane Perturbation and Skin Irritation, (n.d.).

http://journals.plos.org/plosone/article?id=10.1371/journal.pone.0026965 (accessed February 26, 2016).

[19] M. Lukáč, I. Prokipčák, I. Lacko, F. Devínsky, Solubilisation of griseofulvin and rutin in aqueous micellar solutions of gemini and heterogemini surfactants and their mixtures, Eur. J. Pharm. Sci. 44 (2011) 194-199. doi:10.1016/j.ejps.2011.07.011.

[20] A.R. Tehrani-Bagha, R.G. Singh, K. Holmberg, Solubilization of two organic dyes by cationic ester-containing gemini surfactants, J. Colloid Interface Sci. 376 (2012) 112118. doi:10.1016/j.jcis.2012.02.016.

[21] T.-S. Choi, Y. Shimizu, H. Shirai, K. Hamada, Solubilization of disperse dyes in cationic gemini surfactant micelles, Dyes Pigments. 45 (2000) 145-152. doi:10.1016/S0143-7208(00)00015-2.

[22] T. Dam, J.B.F.N. Engberts, J. Karthäuser, S. Karaborni, N.M. van Os, Synthesis, surface properties and oil solubilisation capacity of cationic gemini surfactants, Colloids Surf. Physicochem. Eng. Asp. 118 (1996) 41-49. doi:10.1016/09277757(95)03383-1.

[23] E. Stefanutti, F. Papacci, S. Sennato, C. Bombelli, I. Viola, A. Bonincontro, et al., Cationic liposomes formulated with DMPC and a gemini surfactant traverse the cell membrane without causing a significant bio-damage, Biochim. Biophys. Acta BBA Biomembr. 1838 (2014) 2646-2655. doi:10.1016/j.bbamem.2014.05.026.

[24] M. El Achouri, M.R. Infante, F. Izquierdo, S. Kertit, H.M. Gouttaya, B. Nciri, Synthesis of some cationic gemini surfactants and their inhibitive effect on iron corrosion in hydrochloric acid medium, Corros. Sci. 43 (2001) 19-35. doi:10.1016/S0010-938X(00)00063-9.

[25] M.S. Kamal, A Review of Gemini Surfactants: Potential Application in Enhanced Oil Recovery, J. Surfactants Deterg. 19 (2015) 223-236. doi:10.1007/s11743-015-17765.

[26] M. Dreja, B. Tieke, Polymerization of Styrene in Ternary Microemulsion Using Cationic Gemini Surfactants, Langmuir. 14 (1998) 800-807. doi:10.1021/la9710738.

[27] M. Dreja, W. Pyckhout-Hintzen, H. Mays, B. Tieke, Cationic Gemini Surfactants with Oligo(oxyethylene) Spacer Groups and Their Use in the Polymerization of Styrene in Ternary Microemulsion, Langmuir. 15 (1999) 391-399. doi:10.1021/la981354v.

[28] T.-S. Choi, Y. Shimizu, H. Shirai, K. Hamada, Disperse dyeing of polyester fiber using gemini surfactants containing ammonium cations as auxiliaries, Dyes Pigments. 50 (2001) 55-65. doi:10.1016/S0143-7208(01)00033-X.

[29] T.-S. Choi, Y. Shimizu, H. Shirai, K. Hamada, Disperse dyeing of nylon 6 fiber using gemini surfactants containing ammonium cations as auxiliaries, Dyes Pigments. 48 (2001) 217-226. doi:10.1016/S0143-7208(00)00105-4.

[30] V. Seredyuk, K. Holmberg, Stabilization of Latex by Heterogemini Surfactants, J. Colloid Interface Sci. 241 (2001) 524-526. doi:10.1006/jcis.2001.7745.

[31] K. Chen, D.C. Locke, T. Maldacker, J.-L. Lin, S. Aawasiripong, U. Schurrath, Separation of ergot alkaloids by micellar electrokinetic capillary chromatography using cationic Gemini surfactants, J. Chromatogr. A. 822 (1998) 281-290. doi:10.1016/S0021-9673(98)00672-4. 
[32] R. Oda, I. Huc, S.J. Candau, Gemini Surfactants as New, Low Molecular Weight Gelators of Organic Solvents and Water, Angew. Chem. Int. Ed. 37 (1998). doi:10.1002/(SICI)1521-3773(19981016)37:19<2689::AID-ANIE2689>3.0.CO;2-Z.

[33] K. Czechura, A. Sayari, Synthesis of MCM-48 Silica Using a Gemini Surfactant with a Rigid Spacer, Chem. Mater. 18 (2006) 4147-4150. doi:10.1021/cm060265y.

[34] A. Masuyama, C. Endo, S. Takeda, M. Nojima, D. Ono, T. Takeda, Ozone-Cleavable Gemini Surfactants. Their Surface-Active Properties, Ozonolysis, and Biodegradability, Langmuir. 16 (2000) 368-373. doi:10.1021/la9907578.

[35] T. Banno, K. Kawada, S. Matsumura, Creation of Novel Green and Sustainable Gemini-Type Cationics Containing Carbonate Linkages, J. Surfactants Deterg. 13 (2010) 387-398. doi:10.1007/s11743-010-1224-5.

[36] F. Devínsky, L. Masárová, I. Lacko, Surface activity and micelle formation of some new bisquaternary ammonium salts, J. Colloid Interface Sci. 105 (1985) 235-239. doi:10.1016/0021-9797(85)90363-7.

[37] F. Devínsky, I. Lacko, F. Bittererová, L. Tomečková, Relationship between structure, surface activity, and micelle formation of some new bisquaternary isosteres of 1,5pentanediammonium dibromides, J. Colloid Interface Sci. 114 (1986) 314-322. doi:10.1016/0021-9797(86)90417-0.

[38] F. Devínsky, I. Lacko, T. Imam, Relationship between structure and solubilization properties of some bisquaternary ammonium amphiphiles, J. Colloid Interface Sci. 143 (1991) 336-342. doi:10.1016/0021-9797(91)90267-C.

[39] R. Zana, M. Benrraou, R. Rueff, Alkanediyl-.alpha.,.omega.bis(dimethylalkylammonium bromide) surfactants. 1. Effect of the spacer chain length on the critical micelle concentration and micelle ionization degree, Langmuir. 7 (1991) 1072-1075. doi:10.1021/la00054a008.

[40] E. Alami, G. Beinert, P. Marie, R. Zana, Alkanediyl-.alpha.,.omega.bis(dimethylalkylammonium bromide) surfactants. 3. Behavior at the air-water interface, Langmuir. 9 (1993) 1465-1467. doi:10.1021/la00030a006.

[41] M. Pisárčik, M.J. Rosen, M. Polakovičová, F. Devínsky, I. Lacko, Area per surfactant molecule values of gemini surfactants at the liquid-hydrophobic solid interface, J. Colloid Interface Sci. 289 (2005) 560-565. doi:10.1016/j.jcis.2005.03.092.

[42] H. Hirata, N. Hattori, M. Ishida, H. Okabayashi, M. Frusaka, R. Zana, Small-Angle Neutron-Scattering Study of Bis(quaternary ammonium bromide) Surfactant Micelles in Water. Effect of the Spacer Chain Length on Micellar Structure, J. Phys. Chem. 99 (1995) 17778-17784. doi:10.1021/j100050a017.

[43] N. Hattori, H. Hirata, H. Okabayashi, M. Furusaka, C.J. O’Connor, R. Zana, Smallangle neutron-scattering study of bis(quaternaryammonium bromide) surfactant micelles in water. Effect of the long spacer chain on micellar structure, Colloid Polym. Sci. 277 (1999) 95-100. doi:10.1007/s003960050373.

[44] M. Pisárčik, F. Devínsky, I. Lacko, Aggregation number of alkanediyl- $\alpha, \omega-$ bis(dimethylalkylammonium bromide) surfactants determined by static light scattering, Colloids Surf. Physicochem. Eng. Asp. 172 (2000) 139-144. doi:10.1016/S0927-7757(00)00589-6.

[45] F. Devínsky, I. Lacko, D. Mlynarčík, V. Račanský, L. Krasnec, Relationship between critical micelle concentrations and minimum inhibitory concentrations for some nonaromatic quaternary ammonium salts and amine oxides, Tenside Deterg. 22 (1985) $10-15$.

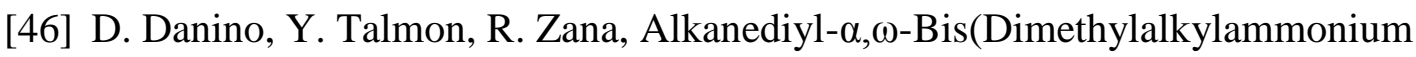
Bromide) Surfactants (Dimeric Surfactants). 5. Aggregation and Microstructure in Aqueous Solutions, Langmuir. 11 (1995) 1448-1456. doi:10.1021/la00005a008. 
[47] UNDECYL ISOCYANATE, Org. Synth. 24 (1944) 94. doi:10.15227/orgsyn.024.0094.

[48] A.A. Belyaev, V.F. Gopko, L.B. Radina, N.M. Peretolchina, Z.P. Sof'ina, G.M. Anoshina, et al., Nitrosoalkylureas derived from alkylammonium salts and their antitumor activity, Pharm. Chem. J. 20 (1986) 306-311. doi:10.1007/BF00758608.

[49] M.J. Rosen, Surfactants and Interfacial Phenomena, 3rd ed., J. Wiley \& Sons, New York, 2004.

[50] R. Zana, J. Xia, Gemini Surfactants: Synthesis, Interfacial and Solution-Phase Behavior, and Applications, CRC Press, 2003.

[51] S.W. Provencher, Constrained regularization method for inverting data represented by linear algebraic or integral equations, Comput. Phys. Commun. 27 (1982) 213-227.

[52] R. Zana, Surfactant Solutions, New Methods of Investigation, Marcel Dekker Inc., New York and Basel, 1987.

[53] J.-P. Hansen, J.B. Hayter, A rescaled MSA structure factor for dilute charged colloidal dispersions, Mol. Phys. 46 (1982) 651-656. doi:10.1080/00268978200101471.

[54] J.S. Pedersen, D. Posselt, K. Mortensen, Analytical treatment of the resolution function for small-angle scattering, J. Appl. Crystallogr. 23 (1990) 321-333. doi:10.1107/S0021889890003946.

[55] M. Lukáč, I. Lacko, M. Bukovský, Z. Kyselová, J. Karlovská, B. Horváth, et al., Synthesis and antimicrobial activity of a series of optically active quaternary ammonium salts derived from phenylalanine, Cent. Eur. J. Chem. 8 (2010) 194-201. doi:10.2478/s11532-009-0126-8.

[56] M. Lukáč, M. Mrva, M. Garajová, G. Mojžišová, L. Varinská, J. Mojžiš, et al., Synthesis, self-aggregation and biological properties of alkylphosphocholine and alkylphosphohomocholine derivatives of cetyltrimethylammonium bromide, cetylpyridinium bromide, benzalkonium bromide $(\mathrm{C} 16)$ and benzethonium chloride, Eur. J. Med. Chem. 66 (2013) 46-55. doi:10.1016/j.ejmech.2013.05.033.

[57] R. Zana, Ionization of cationic micelles: Effect of the detergent structure, J. Colloid Interface Sci. 78 (1980) 330-337. doi:10.1016/0021-9797(80)90571-8.

[58] C. Tanford, Micelle shape and size, J. Phys. Chem. 76 (1972) 3020-3024. doi:10.1021/j100665a018.

[59] L.M. Bergström, V.M. Garamus, Geometrical Shape of Micelles Formed by Cationic Dimeric Surfactants Determined with Small-Angle Neutron Scattering, Langmuir. 28 (2012) 9311-9321. doi:10.1021/la301190d.

[60] R. Sabaté, M. Gallardo, J. Estelrich, Electrophoretic properties of dodecyltrimethylammonium bromide micelles in $\mathrm{KBr}$ solution, Electrophoresis. 21 (2000) 481-485. doi:10.1002/(SICI)1522-2683(20000201)21:3<481::AIDELPS481>3.0.CO;2-9.

[61] S.J. Candau, E. Hirsch, R. Zana, New aspects of the behaviour of alkyltrimethylammonium bromide micelles : light scattering and viscosimetric studies, J. Phys. 45 (1984) 1263-1270. doi:10.1051/jphys:019840045070126300.

[62] A. Chojecka, O. Wiercińska, E. Röhm-Rodowald, K. Kanclerski, B. Jakimiak, Susceptibility of selected strains used for evaluation of biocidal efficiency of disinfectants and antibiotic-resistant strains to didecyldimethyammonium chloride in 2-propanol, Med. Dośw. Mikrobiol. 67 (2015) 47-53.

[63] P. Balgavý, F. Devínsky, Cut-off effects in biological activities of surfactants, Adv. Colloid Interface Sci. 66 (1996) 23-63. doi:10.1016/0001-8686(96)00295-3. 
[64] A.K. Ghumare, B.V. Pawar, S.S. Bhagwat, Synthesis and Antibacterial Activity of Novel Amido-Amine-Based Cationic Gemini Surfactants, J. Surfactants Deterg. 16 (2012) 85-93. doi:10.1007/s11743-012-1406-4.

[65] N.A.R. Gow, F.L. van de Veerdonk, A.J.P. Brown, M.G. Netea, Candida albicans morphogenesis and host defence: discriminating invasion from colonization, Nat. Rev. Microbiol. (2011). doi:10.1038/nrmicro2711.

[66] T.J. Silhavy, N. Ruiz, D. Kahne, Advances in understanding bacterial outermembrane biogenesis, Nat. Rev. Microbiol. 4 (2006) 57-66. doi:10.1038/nrmicro1322. 


\section{Figure captions}

Fig. 1. Dependence of surface tension on log surfactant concentration for gemini surfactants $\mathbf{u 2}, \mathbf{u 4}, \mathbf{u 6}$.

Fig. 2. Dependence of surface tension on log surfactant concentration for gemini surfactants $\mathbf{u} 8, \mathbf{u 1 0}$.

Fig. 3. cmc plotted as a function of $\mathbf{u}(\mathbf{s})$ gemini surfactant spacer length s. cmc of 12-s-12 gemini surfactants [39] is indicated by open circles.

Fig. 4. Area per gemini molecule $A$ at the air/water interface plotted as a function of $\mathbf{u}(\mathbf{s})$ gemini surfactant spacer length s. A of 12-s-12 gemini surfactants [22] is indicated by solid circles.

Fig. 5. Schematic representation of the arrangement of $\mathbf{u} 8$ gemini molecules at the air/water interface.

Fig. 6. Hydrodynamic size of gemini micelles $\mathbf{u} 2, \mathbf{u 4}, \mathbf{u} 6$ plotted as a function of surfactant concentration.

Fig. 7. Hydrodynamic size of gemini micelles $\mathbf{u 8}, \mathbf{u 1 0}$ plotted as a function of surfactant concentration.

Fig. 8. Hydrodynamic size of $\mathbf{u}(\mathbf{s})$ gemini micelles plotted as a function of surfactant spacer length s at the surfactant concentration $10 \mathrm{x}$ cmc.

Fig. 9. Zeta potential of $\mathbf{u ( s )}$ gemini micelles plotted as a function of surfactant concentration.

Fig. 10. Zeta potential of $\mathbf{u}(\mathbf{s})$ gemini micelles plotted as a function of surfactant spacer length $\mathrm{s}$ at the surfactant concentration $5 \mathrm{x}$ cmc.

Fig. 11. Micelle aggregation number of $\mathbf{u}(\mathbf{s})$ gemini surfactants plotted as a function of surfactant concentration.

Fig. 12. Slope values of concentration dependences of the $\mathbf{u}(\mathbf{s})$ micelle aggregation number plotted as a function of surfactant spacer length $\mathrm{s}$.

Fig. 13. Micelle aggregation number of $\mathbf{u}(\mathbf{s})$ gemini surfactants plotted as a function of surfactant spacer length s for all investigated concentrations.

Fig. 14. SANS scattering curves for micellar solutions of $\mathbf{u} \mathbf{2}, \mathbf{u} \mathbf{6}$ and $\mathbf{u} \mathbf{1 0}$ surfactants at concentrations of 2, 4 and $10 \mathrm{wt} \%$ in heavy water.

Fig. 15. Comparison of aggregation number of $\mathbf{u} \mathbf{2}, \mathbf{u} 6$ and $\mathbf{u} 10$ micelles calculated from small angle neutron scattering (SANS) and time-resolved fluorescence quenching experiments (TRFQ).

Fig. 16. Plot of the logarithm of inverse minimum bactericidal concentration of $\mathbf{u}(\mathbf{s})$ gemini surfactants plotted as a function of surfactant spacer length s. The last two points in the plot are the reference compounds. 